\title{
Modelling of Change Propagation to Support the Project Management of a Large-Scale Engineering System
}

\author{
Duseok JEONG ${ }^{1}$, Haruya KAMIYAMA and Kazuhiro AOYAMA \\ Dep. of Systems Inovation, School of Engineering, The University of Tokyo, Japan
}

\begin{abstract}
This research aims to support the engineering change management for a large scale engineering system from the perspective of project management. To achieve the goal, we suggests a method to systemize the tacit process of the skilled managers by using a network-based information model. The model assumed that the direction of the propagation is changeable by engineer's decision. It enables to explore various propagation paths in accordance with the current project status. The explored paths are evaluated from the project management perspective. Although further study requires, we verified the effectiveness of the proposed method on a case study of an FPSO.
\end{abstract}

Keywords. Engineering Change, Change Propagation, Project Management, Decision Support

\section{Introduction}

Most of artefact systems in recent are not developed from scratch. Their design should be modified or improved to satisfy new requirements. Those changes are also required for error corrections, and requirement changes. Likewise, the design of artefact system is often changed across whole development process. To change design is considered as one of the most powerful driving force and unavoidable work in system development [1]. Many researchers have used various terms mixed for this behaviour; engineering change, design change, and simply change[2]. This research used the term of 'Engineering change' (EC) and defined this term as changes to the constituent elements of a system (Ex: parts drawing, parameter set-up, embedded software, etc.) that have already been released, regardless of the scale. Many companies make an effort continuously for the engineering change management[3]. Meanwhile, product complexity has steadily increased in all domains of engineering[4], so that an EC in one part has become more likely to lead to ECs in other parts. This phenomenon is also known as change propagation.

The propagation might result in undesired ECs which have an adverse effect on the downstream developing or manufacturing. Clarkson et al. suggest that propagation is an important factor to predict the impact of an EC[1]. In case of large-scale engineering systems such as ships, naval architectures, or skycrappers, various project elements(e.g. outsourcing, construction, installation) are concurrently conducted and intricately

\footnotetext{
${ }^{1}$ Corresponding Author, Email: jeong@m.sys.t.u-tokyo.ac.jp.
} 
intertwined. Available Design options for the EC and it impacts depend on when the EC is conducted. Thus, it requires the consideration for the status of the project progress as well as engineering things. However, few studies consider the EC from the perspective of project management, while many previous studies provide the understanding into the effect of EC on an engineering system. Experienced managers can make an efficient and effective EC plan based on their understaing of the project progress status and the system structure. Those processes strongly depend on the individual ability and remain tacit in many cases. Therefore, this research suggests a method to systemize the process by using a network-based information model that enables to express propagation path intuitively. The proposed propagation model assumed that the propagation direction is determined by the engineer's decision, therefore, changeable. This enables to explore various propagation paths in accordance with the current project status. The explored paths are evaluated from the project management perspectives: Quality, Cost, and Delivery. Consquently, the proposed method is expected to help to make an EC plan without relying on the tacit knowledge.

\section{Modelling method of system development project}

The proposed method employs a network-based model to explore change propagation paths and evaluate their risk on the development project. The advantage of network representation is to assess indirect linkage [5]. It helps to comprehend propagation paths easily and intuitively. As depicted in Figure 1, system structure is described as a network of three elements: Design Parameter, Constraint, and Component. And, task network enables to consider EC from a perspective of schedule. This section explains the underlying structure of the proposed method.

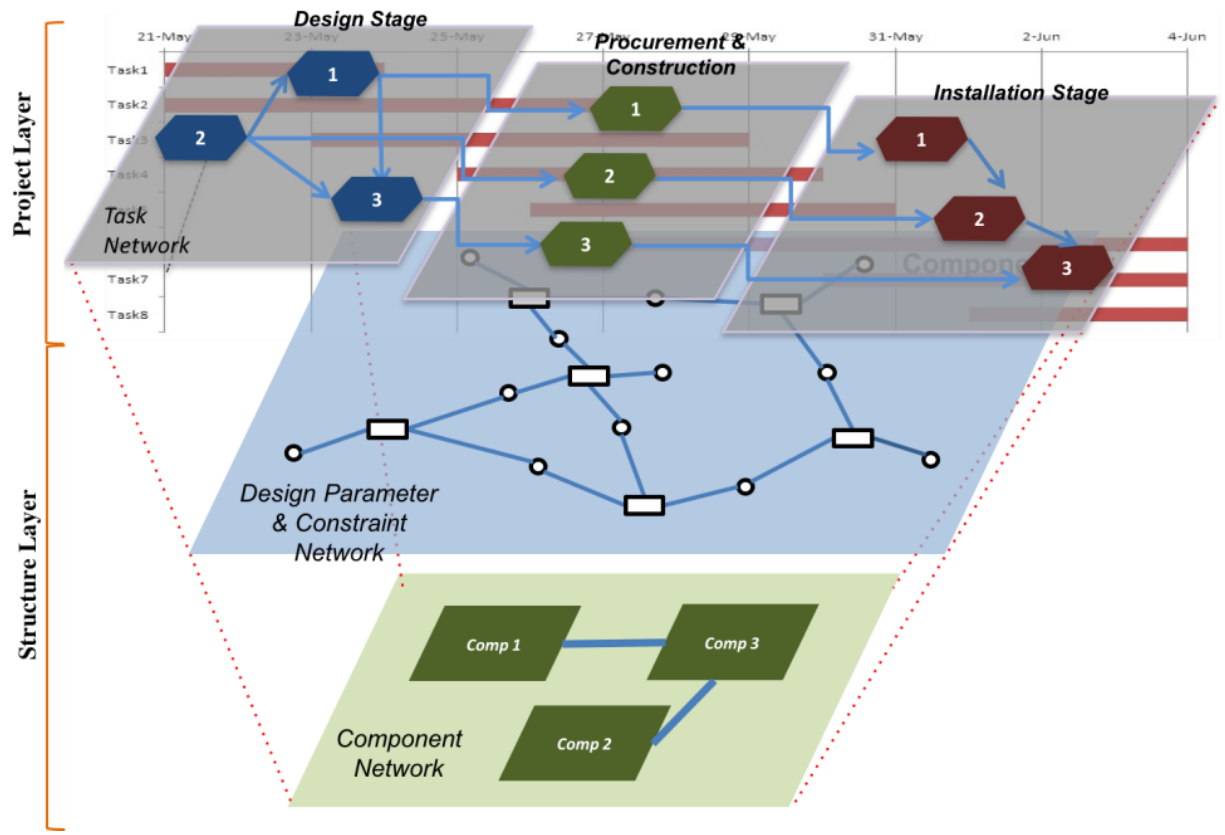

Figure 1. Overview of the proposed modelling method. 


\subsection{Component}

The term 'component' in this research refers to a physical object constituting an engineering system such as a part or a module. Each component is assumed a functionally complete object, so that they could not be decomposed or modularized further. Therefore, the hierarchy structure of components is not considered in this model, unlike other product modeling methods. A component is described as a set of DPs, thus, each component contains at least one design parameter. Each component has a cost estimate(10 levels) for the evaluation of engineering change plan.

\subsection{Design Parameter (DP)}

DPs represent the functional and the physical characteristic of a component. Thus, they are considered as the main object of engineering design behaviour. This research identified two types of the DP according to their properties: attribute and requirement. Attribute DPs describe a product system objectively such as a physical specification or the measure of functional behaviour. Weight, size, and velocity are good examples of attribute DPs.

On the other hand, requirement DPs are related to the subjective aspect of a system. There are various design requirements that should be satisfied in order to operate a system properly as purposed. Those requirements come from both inside (e.g. spatial layout, the characteristic of the material, and sort of dynamics) and outside (e.g. legislation, interface standard, and customer demand) of a system. Requirement DPs translate the user needs into engineering terms. Conditional parameters such as the maximum speed and the minimum strength are considered as requirement DPs.

\subsection{Constraint}

The concept of nondirectional relationships between DPs is suggested by Yoshikawa and Minami [6]. Based on that concept, Koga and Aoyama suggest the notion of constraint that describes the concrete relationships between DPs, e.g, mathematical formula, chart, or semantic relationship [7]. DPs are interrelated in accordance with product design and realise a product system. In most case, it is impossible to determine each DP independently regardless of their connectivity. Thus, it needs to consider the related DPs concurrently, namely, they constrain each others' change. This research adopted the term 'Constraint' referring to a relationship among a set of those interconstrained DPs, to explore the propagation path.

Following shows how to build a product model by using the notion of the DP and the constraint. Equation 1 and 2 represent a part of an engine physics and its design requirement respectively. It is possible to achieve these kinds of information from the existing engineering supporting tools as well as the knowledge of an engineer.

$$
\begin{array}{rlrl}
P_{b} & = & & \text { cylinder mean pressure } \\
T=\frac{1}{4 \pi}\left(P_{b} \cdot \Delta x \cdot A_{t}\right) \quad & \text { where } \Delta x & & \text { piston stroke } \\
A_{t} & = & \text { piston area }
\end{array}
$$

$T \geq$ minimum torque 
Equation 1 consists of four variables: torque, cylinder mean pressure, piston stroke and piston area. They are considered as attribute DPs that describe the physics of an engine. On the other hand, the constant in equation 1 is not considered as a DP since they are not adjustable. The aim of equation 1 is to determine the torque, and DPs in the equation is considered to be related to the aim. Thus, the DPs are bound as a one network form by a constraint. In equation 2 , minimum torque is considered as a requirement DP and torque is an attribute DP as in equation 1. In the same way of equation 1 , equation 2 is also described as a network form as figure 2 depicts. The relation among DPs that are required to consider concurrently is bound by a constraint, regardless of an equation or inequation. And it is possible to easily integrate via the shared DP. This enables to build a model even without the interdisciplinary understanding of a system.

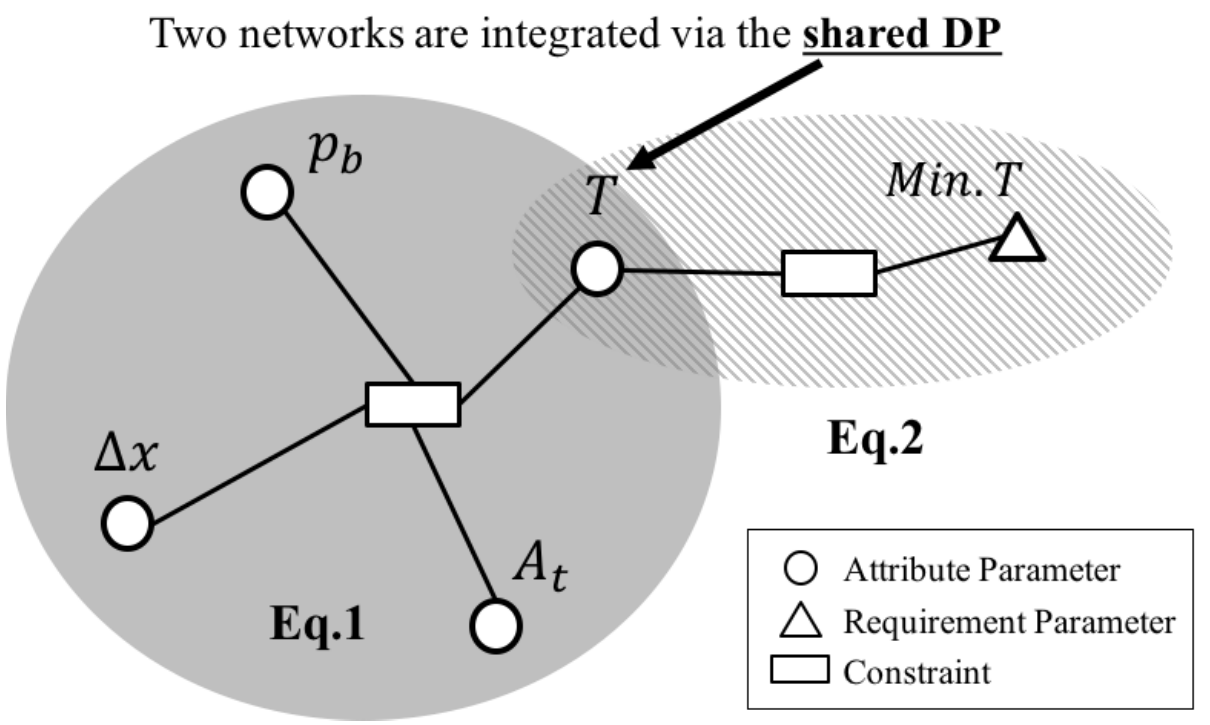

Figure 2. Example of DP and Constraint modelling from equations.

\subsection{Task}

The term 'task' in this research refers to a unit of engineering behavior. Tasks can be classified into several stages by their implementation timing, e.g. concept design, detail design, procurement/construction, and installation. The number and classification of the stages are determined by the engineer and can vary by project. Tasks are connected with other tasks according to the development process so that the development process is described as a network form. The engineering information is embodied and implemented in real things with the development stage progresses. Thus, it should be avoided for ECs in downstream stages, that might lead to a bigger impact on the delivery. Task is also described as a set of DPs with approximate duration data(10 levels). 


\section{Change propagation and engineering change plan (ECP)}

This research defined an engineering solution to the change request, as 'Engineering Change Plan (ECP).' As previously mentioned, this research considered that change propagation occurs as a result of an engineering behavior in the process of an EC. It is rarely possible to adjust directly the functional aspect of a product system for satisfying change request. Thus, it should be to adjust other adjustable DPs that can lead to an effect on the objective of an EC. This is considred to propagate change effect intentionally to satisfy the request.

Assume a case in which piston area in the model of equation 1 is changed but torque is not allowed to change as figure 3 depicts. The change of the area leads to the change of the torque via a causal relationship between them. However, it is possible to change the area with maintaining the torque by adjusting the other DPs: cylinder mean pressure and piston stroke. Consequently, the direction of this propagation will be changed from the torque to the chosen DP, and the torque is maintained.

Meanwhile, the dependencies within a product structure make the change effect to propagate unintentionally. It usually occurs in a causal relationship by the law of physics. Unintentional propagation has a risk to lead engineering failure. Thus, these engineering choices are iterated until the plan satisfy the change request with minimum adverse effect. ECP is described by the collection of included DPs and constraints. However, it is still unknown which design option is better than others at this point. Therefore, ECPs require being evaluated on perspectives of project management.

3. To restore the constraint with maintaining torque, other DPs $\left(p_{b}, A_{t}\right.$, both or neither) are considered as an

2. Constraint 1 consisting of $p_{b}, \Delta x, A_{t}$ and $\mathrm{T}$ become uncertain condition design option.

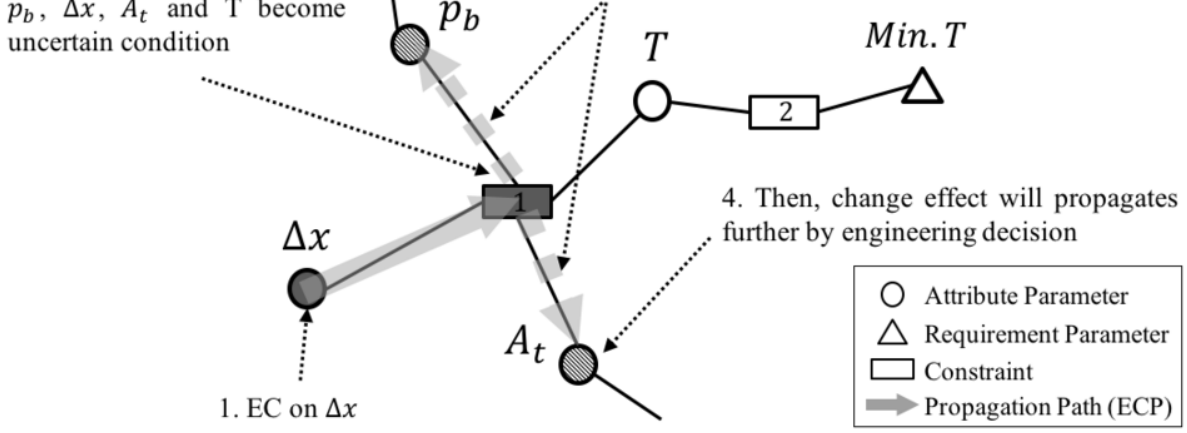

Figure 3. Exploring an ECP with change propagation.

\section{Evaluation of the plans}

Engineering change requires resources as if other development behavior: cost and work hour. In addition, the plan should satisfy the change requests without any conflict of other requirements. This section proposes the evaluation indices of those perspectives for the project management. And the evaluation result of the indices is described as risk level, not specific score, because the proposed model is based on highly abstracted 
data. Accordingly, the result only suggests the comparative superiority between engineering change plans.

At first we made following assumptions to predict approximately required resources for an EC.

1. The more elements are changed, the more resources are required

2. The more important element is changed, the more resources are required

3. The later change, the more resources are required

Based on these assumptions, equation 3 and 4 represent Cost Increase Risk (CIR) and Schedule Delay Risk (SDR) respectively. As mentioned in assumption 1, both of risk indices is the sum of the risk of each elements involved with an ECP. And change scale means how many DPs in task or component are involved with the plan. This index is calculated from the number of changed DPs divided by total number of DPs in the element. Cost and duration are predetermined information as previously explained. These indices represent the assumption 2: A component with higher cost, and a task with the longer duration can be considered the more important. The assumption 3 is represented by current status of component and task criticality. Current status describes the development stage of the component, and is obtained from the associated task via shared DPs. This index is closely related with sunken cost. An EC in design stage only completed with the drawing change, while an EC in later stage requires huge cost including re-build. Task criticality means how close to project end, and is calculated from the distance between the task node and the end node of task network.

$$
\begin{aligned}
& C I R=\sum_{\text {involvedComps }}(\text { changeScale } \cdot \text { cost } \cdot \text { currentStatus }) \\
& S D R=\sum_{\text {involvedTasks }}(\text { changeScale } \cdot \text { duration } \cdot \text { criticality })
\end{aligned}
$$

Second, all requirements should be satisfied in order to achieve the purposed quality of a system. In an EC process, the intricately connected product structure can lead to an unintentional propagation to other requirements that are not planed in an initial EC. It is called 'Requirement Conflict(RC)' in this research. This propagation might have an adverse effect on the overall performance of a product system. The conflict is assumed to occur in case an ECS includes requirement parameters. Thus, the more requirement DPs involved in the ECS have the more risk of the conflict. As equation 5 and 6, the number of involved DP in an ECP represents RC.

$$
\begin{aligned}
& \mathrm{RCR}=\sum f(x), \forall \mathrm{x} \in \mathrm{ECP} \\
& \mathrm{f}(\mathrm{x})=\left\{\begin{array}{lc}
1, & \text { if } x \in \text { req. } D P S \\
0, & \text { otherwise }
\end{array}\right.
\end{aligned}
$$

\section{Case study: FPSO(Floating, Production, Storage, and Offloading)}

An FPSO has been used for offshore oil production system as alternative technology of fixed platform. They are designed to receive oil from subsea, process them, and store until it can be offloaded to a tanker. FPSOs are particularly effective in remote or deep water location, because they are easy to install and do not require a pipeline infra to 
export oil. We made a simple model of FPSO based on the technical documents. This model consists 6 components and 59 design parameters as figure depicts, each component descriptions are showed in table 1.

By using a prototype system with an FPSO case, we explored 1000 ECPs and evaluated them at three different timings. Plot graph in Figure 4 is visualized the result of evaluation. The closer to the left corner means the better plans. Colors of dot represent the change timing: red(early), green(mid), and blue(late). This can be said that ECs at the earlier timing tend to have the lower risk, as explained in Section 3. Also, it can figure out that the risk drastically increase beyond some point.

Thus, we picked up one plan randomly and compared the evaluation result of the plan at different timing. Thus, we picked up one plan with lower risk at early stage, and compared the evaluation result of the plan at different timing. Figure 5 shows the comparison result of the ECP. The network graphs above are the task network of an FPSO case. Each rectangle represents a task, and the current status of the task is represented by color; Red, orange and green means finished task, on-going task and planned task, respectively. And, rectangles with circle mark are the involved tasks. Compared with the left network, the right shows that tasks are more progressed from the color. The EC at later timing involves three finished tasks and three on-going tasks, while the EC at earlier timing involves only three on-going tasks. It can figure out that later timing have has higher risk on increasing re-work hour. The bar graphs below represent the required cost of the ECP. As mentioned above, the evaluation at later timing has more finished and on-going tasks. This means that there might be sunken cost. Likewise, change at early timing has the merit from the cost aspect.

On the other hands, there are no difference of quality aspect by the index proposed in this paper, because two comparison ECPs consists same DPs. However, the project progress has an effect on the feasibility of adjusting DPs. This might lead to another engineering conflict. The future study requires considering it and improving the quality index.

Consequently, we verified that the impact of the EC is dependent on when it is conducted. This also means that the certain ECP can not always be the best option. Engineers require considering the project aspect as well as the engineering aspect in order to make the best change plan.

Table 1. Component decription of an FPSO.

\begin{tabular}{|c|c|}
\hline Component & Description \\
\hline Hull & $\begin{array}{l}\text { The watertight body of an FPSO fully covered with a deck. Atop the deck is } \\
\text { a production structures. }\end{array}$ \\
\hline Turret System & $\begin{array}{l}\text { Enables FPSOs to position the vessel favourably against the wind, current } \\
\text { and wave, with delivering crude oil from sea bed. }\end{array}$ \\
\hline Crude Separation & Separates gas, oil, and water from crude by using their different densities. \\
\hline Gas Compressor & Adjust the gas pressure to inject to oil reservoir. \\
\hline Water Treatment System & Removes sulfates and other unwanted elements from injection water. \\
\hline Power Generator & $\begin{array}{l}\text { Supplies electricity for operation to production structures and seabed } \\
\text { structures }\end{array}$ \\
\hline
\end{tabular}




\section{Conclusion}

ECs are usually considered as unavoidable works in development process. However, their propagative characteristic is still not understood thoroughly. The development of a large-scale system requires considering the complex structure of the system as well as the project progress in which external factors of a system are intricately intertwined. This makes ECs hard to understand and time-consuming process. Hence, the EC process still strongly depends on the individual ability and remain tacit knowledge.

This research suggests a method to systemize the processes by using a networkbased modelling method. The model assumed that DPs are constrained each other to be stable, in accordance with engineering intention. Change propagation is caused in case that the constraint is broken by engineering behaviour. This is explained by the notion of the constraint proposed. By using the feature, the model enables to explore various feasible propagation paths through system structure. The explored paths are evaluated by the proposed indices that represent the project management perspective. It is expected to help to clarify the change impact and make an EC plan without relying on the tacit knowledge. Also we could verify that EC processes are also affected from external factors such as project progress.

Although further studies require, we verified the effectiveness of the proposed method on the case study of an FPSO project. Now we are considering other applications of the proposed modelling method. One of them is to support design for upgradability that enables a long-life cycle system to operate properly with operational environment changes.

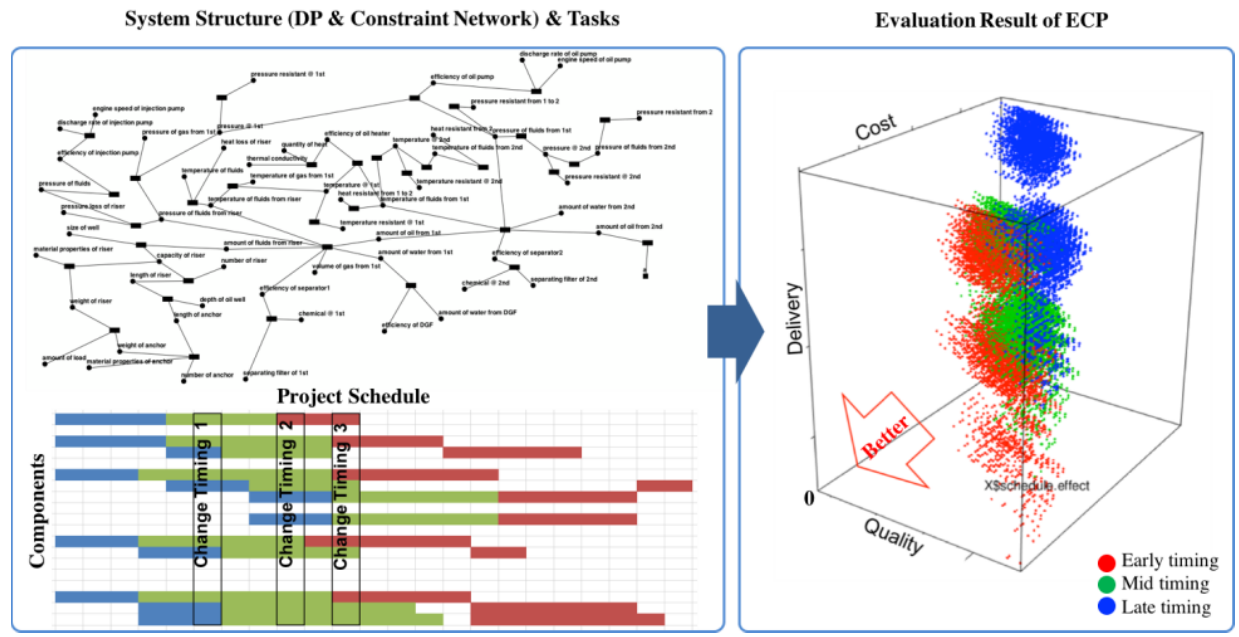

Figure 4. An FPSO model and the evaluation result of the plans. 


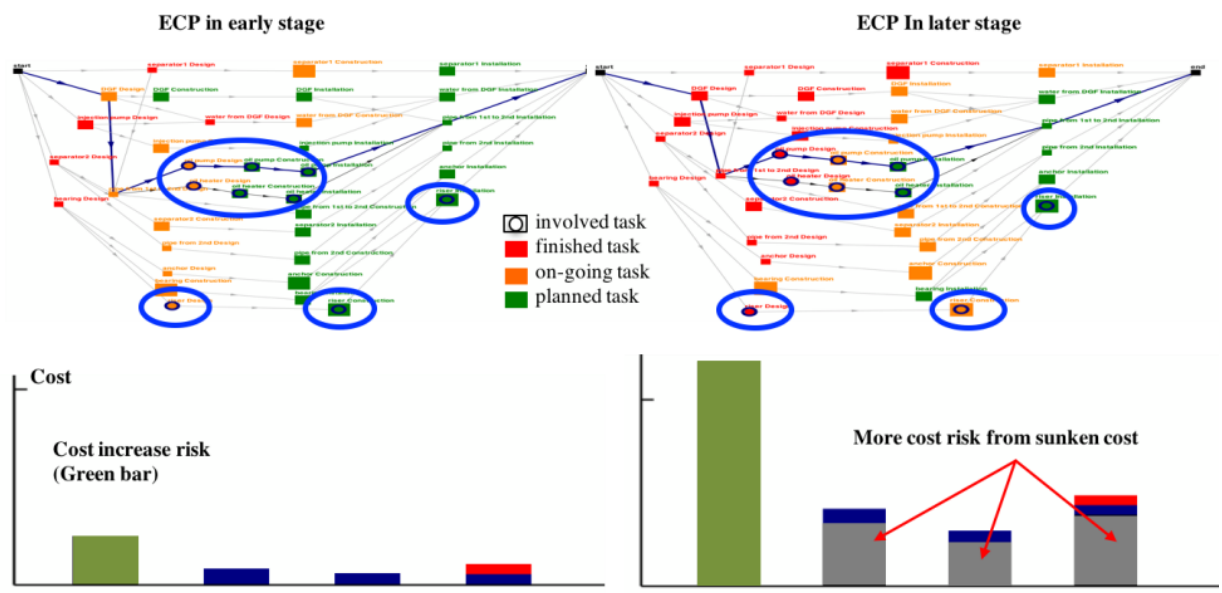

Figure 5. The comparison of the evaluation result by change timing.

\section{Reference}

[1] P.J. Clarkson et al., Predicting change propagation in complex design, In: Proceedings of ASME design engineering technical conferences, Pittsburgh, USA, 2001, CD-ROM, paper no. DETC2001/DTM-21698

[2] T.A.W. Jarratt, C.M. Eckert, N.H.M. Caldwell, Engineering change: an overview and perspective on the literature, Res Eng Des, 2011, Vol. 22, pp. 103-124.

[3] E. Fricke, et al., Coping with changes: Causes, findings, and strategies, System Engineering, 2000, Vol. 3, pp. 169-179.

[4] U. Lindermann, M. Maurer and T. Braun, Structural Complexity Management, Springer, Berlin, 2009

[5] M. Ghoniem, J.-D. Fekete and P. Castagliola. A Comparison of the Readability of Graphs Using Node-Link and Matrix-Based Representations, IEEE Symposium on Information Visualization, TX, USA, 2004, pp. 17-24.

[6] S. Yoshikawa and M. Minami, Systematization of Design Problems by Graph Theory, In: Proceedings of the International Design Conference, Dubrovnik, Croatia, 2004, paper no. DS32_028

[7] T. Koga and K. Aoyama, Design Porcess Guide Method for Minimizing Loops and Conflicts, Journal of Advanced Mechanical Design, Systems, and Manufacturing, 2009, Vol. 3, pp. 191-202. 